\title{
Neutron diffraction study of phase transitions and thermal expansion of $\mathrm{SrFeAsF}$
}

\author{
Y. Xiao, ${ }^{1, *}$ Y. Su, ${ }^{2}$ R. Mittal, ${ }^{2,3}$ T. Chatterji, ${ }^{4}$ T. Hansen, ${ }^{5}$ S. Price,,${ }^{1}$ C. M. N. Kumar, ${ }^{1}$ J. Persson, ${ }^{1}$ S. Matsuishi, ${ }^{6}$ Y. Inoue, ${ }^{6}$ \\ H. Hosono, ${ }^{6}$ and Th. Brueckel ${ }^{1,2,4}$ \\ ${ }^{1}$ Institut fuer Festkoerperforschung, Forschungszentrum Juelich, D-52425 Juelich, Germany \\ ${ }^{2}$ Juelich Centre for Neutron Science, IFF, Forschungszentrum Juelich, Outstation at FRM II, Lichtenbergstrasse 1, \\ D-85747 Garching, Germany \\ ${ }^{3}$ Solid State Physics Division, Bhabha Atomic Research Centre, Trombay, Mumbai 400 085, India \\ ${ }^{4}$ Juelich Centre for Neutron Science, IFF, Forschungszentrum Juelich, Outstation at Institut Laue-Langevin, BP 156, \\ 38042 Grenoble Cedex 9, France \\ ${ }^{5}$ Institut Laue-Langevin, BP 156, 38042 Grenoble Cedex 9, France \\ ${ }^{6}$ Frontier Research Center, Tokyo Institute of Technology, 4259 Nagatsuta-cho, Midori-ku, Yokohama 226-8503, Japan
}

(Received 8 December 2009; revised manuscript received 9 March 2010; published 26 March 2010)

\begin{abstract}
The magnetic ordering and crystal structure of iron pnictide $\mathrm{SrFeAsF}$ was investigated by using neutron powder-diffraction method. With decreasing temperature, the tetragonal to orthorhombic phase transition is found at 180(2) $\mathrm{K}$ while the paramagnetic to antiferromagnetic phase-transition set in at 133(3) K. The big difference between structural and magnetic phase transitions in SrFeAsF indicates the weak magnetic exchange coupling between Fe layers. Similar to the parent compound of other iron-pnictide system, the striped Fe magnetism is confirmed in antiferromagnetic phase and the Fe moment of 0.58(6) $\mu_{B}$ aligned along long $a$ axis. Given the fact that long-range antiferromagnetic order can be described by a power law with $\beta=0.124(16)$, $\mathrm{SrFeAsF}$ can be treated as a quasi-two-dimensional Ising system. Thermal expansion of orthorhombic phase of $\mathrm{SrFeAsF}$ is also investigated. Based on the Grüneisen approximation and Debye approximation for internal energy, the volume of SrFeAsF can be well fitted with Debye temperature of 347(5) K. The experimental atomic-displacement parameters for different crystallographic sites in $\mathrm{SrFeAsF}$ are analyzed with Debye model. Thermal expansion analysis for SrFeAsF suggested that the expansion of FeAs layers plays an important role in determining the thermal expansion coefficient.
\end{abstract}

DOI: 10.1103/PhysRevB.81.094523

PACS number(s): 74.70.Xa, 75.25.-j, 65.40.De

High-temperature superconductivity has recently been discovered in carriers doped iron-pnictide compounds. ${ }^{1-4}$ Till now, the highest $T_{c}$ attained is $57.4 \mathrm{~K}$ in the electron-doped "1111" compound $\mathrm{Ca}_{0.4} \mathrm{Nd}_{0.6} \mathrm{FeAsF}$ (Ref. 5) while for the so-called "122" compound the highest $T_{c}$ of $38 \mathrm{~K}$ is reached in the hole-doped $\mathrm{Ba}_{0.6} \mathrm{~K}_{0.4} \mathrm{Fe}_{2} \mathrm{As}_{2}{ }^{4}$ It is generally believed that the superconductivity in iron pnictides is unlikely due to simple electron-phonon coupling, as demonstrated from extensive studies of phonon dynamics. ${ }^{6,7}$ Magnetism seems to play a crucial role in the appearance of superconductivity and AFM spin fluctuations have thus been suggested to be a possible pairing mechanism. ${ }^{8}$

The spin-density-wave (SDW) transition, which is associated with long-range antiferromagnetic (AFM) order of $\mathrm{Fe}$ moments, is shown in undoped FeAs-based compounds. ${ }^{9-12}$ Band-structural calculations suggested that the SDW is driven either by Fermi surface nesting between electron and hole pockets or by the magnetic exchange coupling between local moments. ${ }^{13-16}$ Furthermore, the SDW transition is found to be accompanied by the tetragonal-to-orthorhombic (T-O) structural phase transition in the 122 family and preceded by the T-O transition for the 1111 family. ${ }^{9-12}$ It is generally accepted that the crystal and magnetic structure in undoped FeAs compound are intimately coupled. Theoretical studies suggested that the structural phase transition is driven by the AFM stripe magnetism directly. ${ }^{14,16,17}$ Therefore, it is important to investigate the relationship between structural properties and AFM ordering of the undoped FeAs compound, especially for the 1111 compound, in which the struc- tural phase transition always occurred at higher temperature than SDW transition temperature.

As a new parent nonsuperconducting compound, $\mathrm{SrFeAsF}$ exhibited prominent anomaly in magnetic and electric property measurements. ${ }^{18-20}$ However, detailed studies have not been carried out to investigate the magnetic and structural transformation in SrFeAsF. In this paper, we investigated the structural and magnetic phase transition as well as thermal expansion in $\mathrm{SrFeAsF}$ compound by using the neutron powder-diffraction (NPD) method. Both structural and magnetic phase transitions are clarified; the temperature dependence of atomic-displacement parameters and thermal expansion are well modeled based on the experimental results. The $\mathrm{SrFeAsF}$ polycrystalline sample of $10 \mathrm{~g}$ was synthesized by a solid-state reaction method as described in Ref. 18 with impurity phases $\left(\mathrm{CaF}_{2}\right.$ and $\left.\mathrm{Fe}_{2} \mathrm{O}_{3}\right)$ of less than $1 \%$. The neutron powder-diffraction measurements were performed on highflux powder diffractometer D20 at Institut Laue Langevin (Grenoble, France). A Ge (115) monochromator was used to produce a monochromatic neutron beam of wavelength $1.88 \AA$ A. The sample was loaded in a vanadium sample holder and then installed in the liquid helium cryostat that can generate temperature down to $2 \mathrm{~K}$. The NPD data were first collected at $2 \mathrm{~K}$ and then collected continuously every 1 min while the sample was warmed from 2 to $240 \mathrm{~K}$ at a rate of $0.4 \mathrm{~K} / \mathrm{min}$. Due to the high neutron flux of D20, highquality diffraction pattern that suitable for Rietveld refinement can be obtained within 5 min for our SrFeAsF sample. Therefore, totally 120 data sets between 2 and $240 \mathrm{~K}$ are obtained by integrating raw data over $2 \mathrm{~K}$ temperature range. 

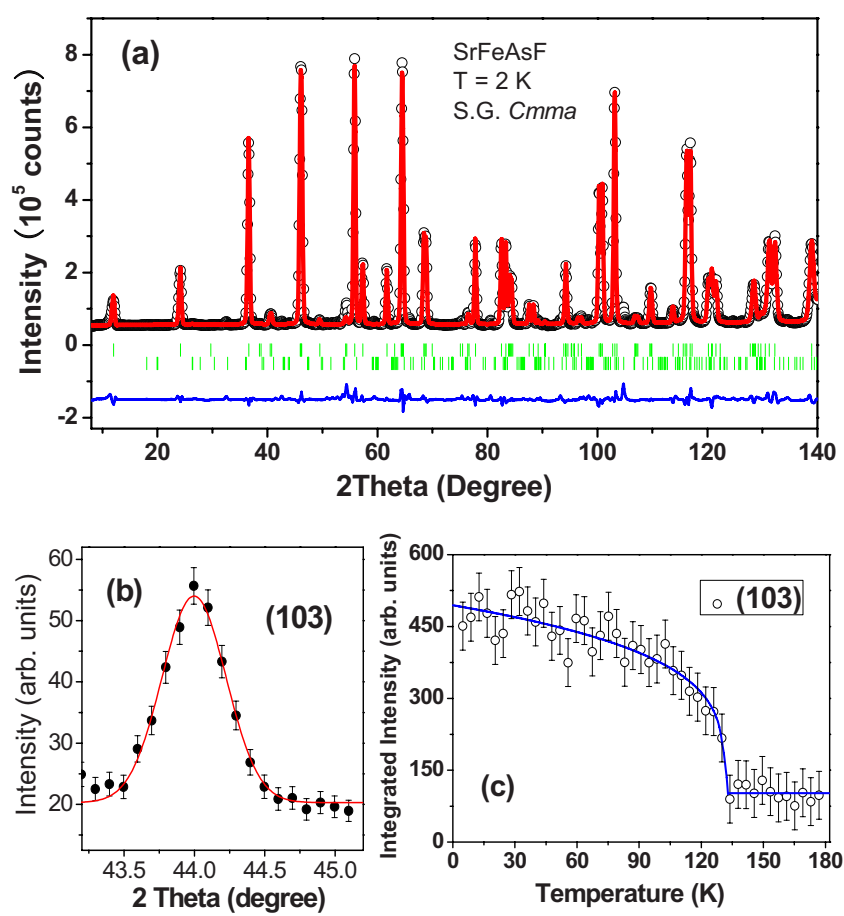

FIG. 1. (Color online) (a) Neutron powder-diffraction pattern of $\mathrm{SrFeAsF}$ at $2 \mathrm{~K}$. The circles represent the observed intensities, the solid line is the calculated pattern. The difference between the observed and calculated intensities is shown at the bottom. The nuclear and magnetic Bragg reflection positions are indicated by the vertical bars at upper and lower positions, respectively. (b) The (103) magnetic reflections obtained by subtracting the NPD pattern measured at $240 \mathrm{~K}$ from the pattern measured at $2 \mathrm{~K}$. (c) Temperature dependence of the integrated intensity of (103) magnetic Bragg reflection. The solid curve is fit to the power law. In order to obtain good statistics, the intensities in panel (c) are obtained by integrating the raw data over $4 \mathrm{~K}$ temperature range.

The program FULLPROF (Ref. 21) was used for the Rietveld refinement of the crystal and the magnetic structures of the compound.

Similar to the CaFeAsF compound, the SrFeAsF also crystallized in orthorhombic structure with space group Cmma at $2 \mathrm{~K}$. Refinement of the neutron-diffraction pattern gave lattice parameters of $a=5.6689(1) \AA, b=5.6260(1) \AA$, and $c=8.9325(2) \AA$ at $2 \mathrm{~K}$. Both lattice parameters and unitcell volume $V$ are larger than that in CaFeAsF, which is consistent with the fact that the atomic radius of $\mathrm{Sr}$ is larger than that of $\mathrm{Ca}$. The refined neutron powder-diffraction pattern of SrFeAsF at $2 \mathrm{~K}$ is shown in Fig. 1(a). Apart from the nuclear Bragg reflections, the magnetic peaks can be fitted by using the same AFM structural model as in $\mathrm{CaFeAsF}$ (Ref. 22) for Fe magnetism and the Fe moment is deduced to be $0.58(6) \mu_{B}$. It seems that the AFM configuration of Fe spins and the small magnitude of moment are a common feature for all FeAs systems. The origin of that small iron moment in these compounds was explained theoretically as the result of the itinerant character of iron spins ${ }^{13}$ or the nearest and next-nearest-neighbor superexchange interactions between $\mathrm{Fe}$ ions which give rise to a frustrated magnetic ground state. ${ }^{14}$ The temperature dependence of integrated intensity of (103) magnetic reflection was plotted in
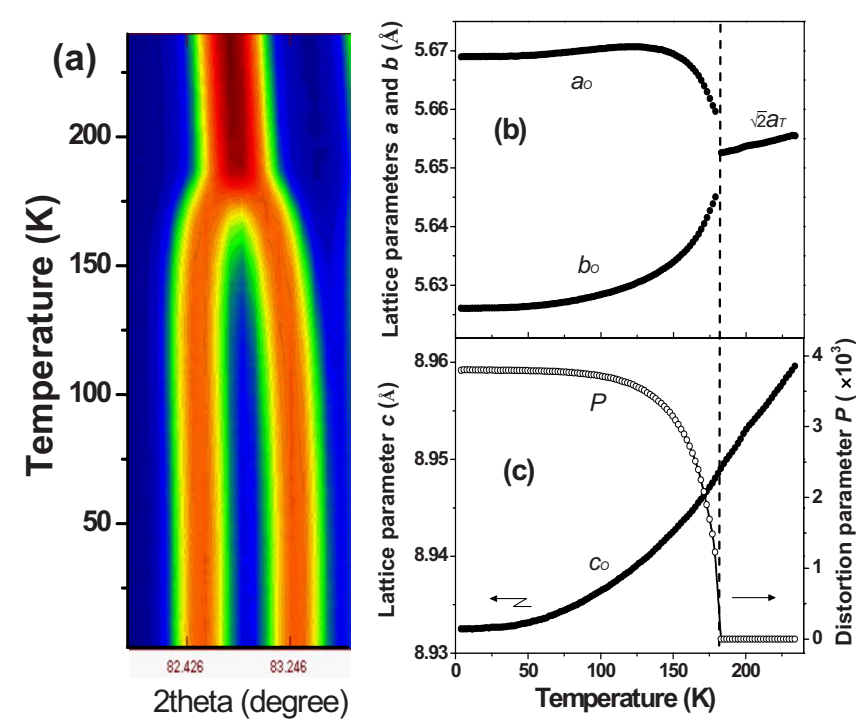

FIG. 2. (Color online) (a) Evolution of $(220)_{T}$ reflections with the change in temperature. The structural phase transition is clearly revealed by the splitting of tetragonal $(220)_{T}$ into orthorhombic $(400)_{O}$ and $(040)_{O}$ reflections. (b) and (c) Temperature dependence of the estimated lattice parameter $a, b$, and $c$ as well as the lattice distortion parameter $P$ for both tetragonal and orthorhombic phases. Dashed line indicates the phase boundary.

Fig. 1(c) to obtain the AFM phase-transition temperature. Fitting the temperature variation in the magnetic order parameter with a power law, $I \propto\left(1-T / T_{N}\right)^{2 \beta}$, yields a critical exponent $\beta$ of $0.124(16)$, as indicated by the solid line in Fig. 1(c). The obtained $\beta$ value is in good agreement with the theoretical value of critical exponent for a two-dimensional Ising model $(\beta=1 / 8)$ (Ref. 23) and is consistent with earlier reports by Baker et al. ${ }^{24}$ The power-law fits in different ranges of reduced temperature below the phase transition lead to small variation in critical exponents, which demonstrated the reliability of fit results and confirmed the twodimensional antiferromagnetism of this fluoropnictide system.

Based on the power-law fit results, the Fe spins are found to order below $T_{N}=133(3) \mathrm{K}$. The onset of the structural phase transition from $P 4 / \mathrm{nmm}$ to Cmma takes place at $T_{S}$ $=180(2) \mathrm{K}$ as indicated by the evolution of $(220)_{T}$ tetragonal reflection with temperature [Fig. 2(a)]. The $T_{S}$ is in accordance with the structural phase transition observed by other macroscopic characterization methods. ${ }^{18-20}$ The variation in lattice parameters can be obtained through the refinement of NPD patterns. As shown in Figs. 2(b) and 2(c), the lattice parameters $a$ and $b$ deviated from each other when the temperature is lower than the structural phase-transition temperature while the lattice parameter $c$ decreased gradually with decrease in temperature. Temperature dependence of structural order parameter, $p=\frac{(a-b)}{(a+b)}$, is also plotted in Fig. 2(c). A continuous phase transition is demonstrated by the smooth evolution of structural order parameter, which is the characteristic of a second-order phase transition. The occurrence of second-order phase transition through $T_{S}$ is also supported by specific-heat measurement for SrFeAsF, where the broad anomaly around $T_{S}$ is observed. ${ }^{19}$ The structural prop- 
erties of SrFeAsF are quite similar to oxypnictide $\mathrm{LaFeAsO},{ }^{9}$ in which two broad second-order transitions are reported corresponding to structural and spin-density-wave transitions, respectively. In contrast to 1111 compounds, both the antiferromagnetic order and structural phase transition occur at same temperature in 122 compounds. As a result, only one pronounced first-order transition is shown in 122 compounds, such as $\mathrm{SrFe}_{2} \mathrm{As}_{2}$ (Ref. 25) and $\mathrm{CaFe}_{2} \mathrm{As}_{2} .{ }^{26}$

The structural distortion from high-symmetry tetragonal phase to low-symmetry orthorhombic phase will result in an inequivalence of nearest- and next-nearest-neighbor coupling $\left(J_{1}\right.$ and $\left.J_{2}\right)$ in FeAs layers and lift the magnetic frustration. The collinear AFM spin configuration is stabilized at the ground state when $J_{2}>J_{1} / 2 .{ }^{14,16}$ Within the Heisenberg magnetic exchange model, it is established that the difference between the structural and AFM transition temperature is strongly correlated with the ratio between out-of-plane magnetic exchange coupling $J_{z}$ and $J_{2} \cdot{ }^{16,27}$ The difference $\left(T_{S}-T_{N}\right) / T_{N}$ will increase with decreasing $J_{z} / J_{2}$ value. For SrFeAsF, the $\left(T_{S}-T_{N}\right) / T_{N}$ is deduced to be $\sim 35 \%$, which is few times greater than that of $\mathrm{LaFeAsO}(\sim 13 \%) .{ }^{9}$ Therefore, it is suggested that the $\mathrm{SrFeAsF}$ possessed small $J_{z} / J_{2}$ value and the magnetic exchange coupling between $\mathrm{Fe}$ layers is rather weak. Given the weak interlayer coupling and the critical behavior of the magnetic order parameter, $\mathrm{SrFeAsF}$ can be well described as a quasi-two-dimensional Ising system. Since spin fluctuations have been suggested to be the driving force of the tetragonal-to-orthorhombic phase transition, the exact nature of spin fluctuations in these phase regimes is still to be established experimentally. SrFeAsF is thus an ideal example for the investigations of the evolution of spin fluctuations from the tetragonal phase to orthorhombic phase via inelastic neutron scattering, owing to the fact of that the difference between the magnetic and structural phase-transition temperatures in $\mathrm{SrFeAsF}$ is the largest among all known iron-pnictide parent compounds. Similar to other iron-pnictide systems, superconductivity can also be realized by doping on $\mathrm{SrFeAsF}$ parent compound with carriers. The $T_{c}$ reported in the $\mathrm{Sm}$-doped $\mathrm{SrFeAsF}$ superconducting compound is $56 \mathrm{~K},{ }^{28}$ among the highest reported for iron pnictides. Actually, superconductivity with $T_{c}$ values between 50 and $57.4 \mathrm{~K}$ is observed in several different 1111 systems. ${ }^{29}$ However, the highest $T_{c}$ obtained for various systems cannot be easily related to either structural or magnetic phase-transition temperature of their parent counterpart. It was revealed that the highest $T_{c}$ of iron pnictide can be reached when $\mathrm{FeAs}_{4}$ lattices forms a regular tetrahedron, ${ }^{29}$ which suggested that the structure perfection of $\mathrm{FeAs}_{4}$ tetrahedron is crucial factor to obtain high- $T_{c}$ superconductor.

The detailed structural information for $\mathrm{SrFeAsF}$ at 2 and $240 \mathrm{~K}$, as obtained from NPD data, are given in Table I. The variation in unit-cell volume with temperature over measured temperature range is shown in Fig. 3. To model the experimental data of the unit-cell volume, we followed the approach of the Grüneisen approximation for the zero-pressure equation of state, in which the effects of thermal expansion are considered to be equivalent to elastic strain. ${ }^{30}$ Thus, the temperature dependence of the volume can be described by $V(T)=\gamma U(T) / K_{0}+V_{0}$, where $\gamma$ is a Grüneisen parameter, $K_{0}$ is the incompressibility and $V_{0}$ is the volume at $T=0 \mathrm{~K}$. By
TABLE I. Refined results of the crystal and magnetic structures for $\mathrm{SrFeAsF}$ at 2 and $240 \mathrm{~K}$. The atomic positions for space group Cmma: $\operatorname{Sr}(4 g)(0,0.25, z), \mathrm{Fe}(4 b)(0.25,0,0.5), \operatorname{As}(4 g)(0,0.25, z)$, and $\mathrm{F}(4 a)(0.25,0,0)$ and for $P 4 / \mathrm{nmm}$ : $\operatorname{Sr}(2 c)(0.25,0.25, z)$, $\mathrm{Fe}(2 b)(0.75,0.25,0.5), \quad \operatorname{As}(2 c)(0.25,0.25, z), \quad$ and $\mathrm{F}(2 a)(0.75,0.25,0)$.

\begin{tabular}{|c|c|c|}
\hline Temperature & $2 \mathrm{~K}$ & $240 \mathrm{~K}$ \\
\hline Space group & Cmma & $P 4 / n m m$ \\
\hline$a(\AA)$ & $5.6689(1)$ & $3.9996(1)$ \\
\hline$b(\AA)$ & $5.6260(1)$ & $3.9996(1)$ \\
\hline$c(\AA)$ & $8.9325(2)$ & $8.9618(4)$ \\
\hline$V\left(\AA^{3}\right)$ & $284.89(2)$ & $143.36(2)$ \\
\hline
\end{tabular}

$\mathrm{Sr}$

$\begin{array}{lcc}z & 0.1584(1) & 0.1583(2) \\ B\left(\AA^{2}\right) & 0.05(4) & 0.44(4)\end{array}$

$\mathrm{Fe}$ $B\left(\AA^{2}\right) \quad 0.04(2) \quad 0.29(2)$

$M_{a}\left(\mu_{B}\right) \quad 0.58(6)$

As

$z \quad 0.6525(1) \quad 0.6515(1)$

$B\left(\AA^{2}\right) \quad 0.05(4) \quad 0.33(4)$

F

$B\left(\AA^{2}\right) \quad 0.13(3) \quad 0.38(3)$

Bond length $(\AA)$

$\mathrm{Fe}-\mathrm{Fe}$

$2.834(2) \times 2$

$2.828(2) \times 4$

Fe-As

$2.813(1) \times 2$

$2.417(2) \times 4$

$2.417(2) \times 4$

Bond angle $\left({ }^{\circ}\right)$

$\mathrm{Fe}-\mathrm{As}-\mathrm{Fe}$

\begin{tabular}{cc}
$71.168(3) \times 2$ & $71.608(3) \times 4$ \\
$71.794(3) \times 2$ & $111.653(3) \times 2$ \\
$111.394(3) \times 2$ & \\
3.43 & 4.98 \\
5.09 & 7.58 \\
3.53 & 5.63 \\
\hline
\end{tabular}

adopting the Debye approximation, the internal energy $U(T)$ is given by

$$
U(T)=9 N k_{B} T\left(\frac{T}{\theta_{D}}\right)^{3} \int_{0}^{\theta_{D} / T} \frac{x^{3}}{e^{x}-1} d x,
$$

where $N$ is the number of atoms in the unit cell, $k_{B}$ is the Boltzmann's constant and $\theta_{D}$ is the Debye temperature. Above model exhibits a good fit to volume variation as indicated by the solid line in Fig. 3. We obtained following physical parameters: $\theta_{D}=347(5) \mathrm{K}, V_{0}=284.89(2) \AA^{3}$, and 


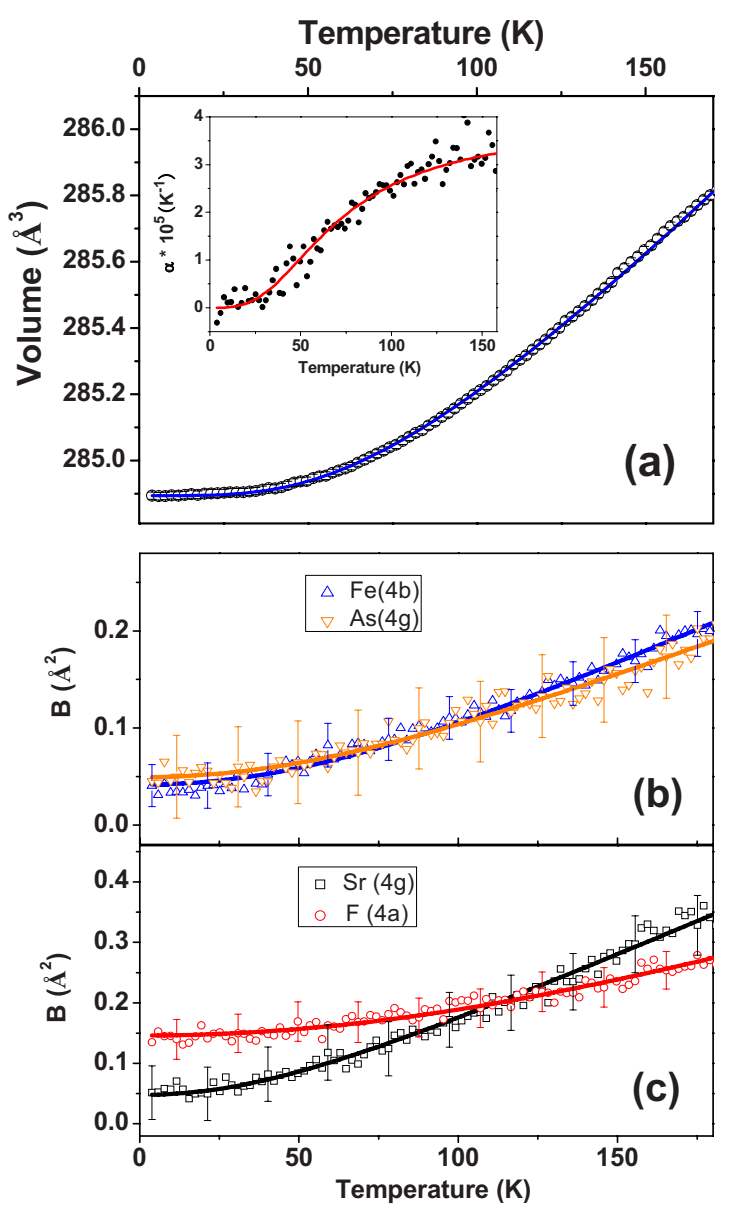

FIG. 3. (Color online) (a) Temperature dependence of the volume of unit cell of SrFeAsF. The solid line is fit of the Debye model as described in the text. The inset shows the thermal-expansion constant of volume as determined from the fit of the Debye model in comparison to the experimental data. (b) and (c) Isotropic thermal parameters for different atomic sites in SrFeAsF.

$\gamma / K_{0}=1.57(3) \times 10^{-9} \mathrm{~Pa}^{-1}$. The value for the Debye temperature as determined from neutron experiments is in reasonable agreement with those determined from specific-heat measurements, $\theta_{D}=339(1) \mathrm{K}$ in Ref. 19. Note that the Debye temperature approximates only the acoustical phonons at low temperature. The thermal-expansion coefficient $\alpha(T)$ which corresponds to the first derivative of $U(T)$ divided by the unit-cell volume at that temperature is plotted in the inset of Fig. 3(a) as the solid dots. The solid curve correspond to the calculated value of $\alpha(T)$ from $V(T)$.

As a basic crystallographic parameter, the atomicdisplacement parameter (ADP) can reflect the atomic thermal motion and provide useful information on the thermal properties of materials. ${ }^{31,32}$ Therefore, the ADPs of different crystallographic sites in $\mathrm{SrFeAsF}$ were also investigated. For the low-temperature Cmma phase, the space-group symmetries of all special positions [ $\mathrm{Sr}(4 g), \mathrm{Fe}(4 b), \mathrm{As}(4 g)$, and $\mathrm{F}(4 a)]$ allow three independent ADP elements. Based on approximate classification, the isotropic $\mathrm{ADP}\left\langle U_{i s o}^{2}\right\rangle$ was applied by assuming that the displacements are the same in all directions. The thermal parameter $B_{\text {iso }}$ which are obtained assuming isotropic thermal motions of atoms are evaluated using the relation $B_{\text {iso }}=8 \pi^{2}\left\langle U_{\text {iso }}^{2}\right\rangle+B_{\text {sta }}$, where $B_{\text {sta }}$ is the static component of the thermal parameter caused by the presence of a certain amount of static disorder in compounds. Figures 3(b) and 3(c) show the temperature variation in experimental thermal parameters for all four atomic sites of SrFeAsF. Debye model was adopted to describe the ADP as a function of temperature

$$
\left\langle U_{i s o}^{2}\right\rangle=\frac{3 \hbar^{2} T}{m k_{B} \theta_{D}^{2}}\left[\Phi\left(\theta_{D} / T\right)+\frac{\theta_{D}}{4 T}\right],
$$

where $\Phi\left(\theta_{D} / T\right)$ is given by

$$
\Phi\left(\theta_{D} / T\right)=\frac{T}{\theta_{D}} \int_{0}^{\theta_{D} / T} \frac{x}{e^{x}-1} d x .
$$

The fitted results for the thermal parameters based on above equations are plotted in Figs. 3(b) and 3(c) as the solid lines in comparison with the experimental results. The Debye temperatures evaluated for different atomic species are 238(6) [Sr], 365(6) [Fe], 348(6) [As], and 611(6) K [F]. The averaged-mass-weighted value for the Debye temperature is obtained to be 332(10) K. The corresponding Debye frequencies for $\mathrm{Sr}, \mathrm{Fe}$, As, and F sites are 4.9(1), 7.6(1), 7.2(1), and 12.7(1) $\mathrm{THz}$ respectively. The small difference between the Debye frequencies for $\mathrm{Fe}$ and As revealed the similar range of phonon frequencies for those two sites. The range of phonon frequencies obtained from our analysis is in very good agreement with our recent inelastic neutron scattering and $a b$ initio phonon-calculation results ${ }^{33}$ for $\mathrm{SrFeAsF}$ indicating the reliability of our analysis.

$A b$ initio electronic-structure calculations for $\mathrm{SrFeAsF}$ (Ref. 34) show that it possessed essentially the same band dispersions in the vicinity of the Fermi surface as in other 1111 compounds, such as $\mathrm{LaFeAsO}$ and $\mathrm{CaFeAsF}$. Furthermore, the electronic states near the Fermi surface are dominated by contributions from $\mathrm{Fe}$ and As, which indicates that the FeAs layers are playing an important role in magnetism and superconductivity of the compound. From our thermalparameter analysis, we also notice that the mean value of the Debye temperature for $\mathrm{Fe}$ and As [355(8) $\mathrm{K}$ ] in $\mathrm{SrFeAsF}$ agrees well with the Debye temperature deduced from the analysis of thermal expansion curve [Fig. 3(a), $\theta_{D}$ $=347(5) \mathrm{K}]$. Therefore, the thermal expansion of FeAs layers is supposed to be the main contribution to the thermal expansion of volume.

In summary, the structural and magnetic phase transition as well as the thermal expansion of $\mathrm{SrFeAsF}$ was investigated by using the neutron-diffraction method. The onset of tetragonal-to-orthorhombic phase transition is at 180(2) K while the paramagnetic to antiferromagnetic phase transition takes place at $133(3) \mathrm{K}$. SrFeAsF exhibits the largest difference between $T_{S}$ and $T_{N}$ among all known iron pnictides, which indicated that the magnetic-exchange coupling between $\mathrm{Fe}$ layers in $\mathrm{SrFeAsF}$ is rather weak than in other iron pnictides. The striped AFM arrangement of Fe spins is confirmed by refinement of neutron data. The magnitude of $\mathrm{Fe}$ moment aligned along $a$ direction is deduced to be $0.58(6) \mu_{B}$. The lattice volume of low-temperature orthorhombic phase can be modeled well in frame of Grüneisen 
approximation with a Debye approximation for the internal energy. The analysis on atomic-displacement parameters of different crystallographic sites suggested that the vibration of
$\mathrm{Fe}$ and $\mathrm{As}$ is coupled in FeAs layers and they contribute mainly to the thermal expansion of volume in SrFeAsF. *y.xiao@fz-juelich.de

${ }^{1}$ Y. Kamihara, T. Watanabe, M. Hirano, and H. Hosono, J. Am. Chem. Soc. 130, 3296 (2008).

${ }^{2}$ X. H. Chen, T. Wu, G. Wu, R. H. Liu, H. Chen, and D. F. Fang, Nature (London) 453, 761 (2008).

${ }^{3}$ H. Takahashi, K. Igawa, K. Arii, Y. Kamihara, M. Hirano, and H. Hosono, Nature (London) 453, 376 (2008).

${ }^{4}$ M. Rotter, M. Tegel, and D. Johrendt, Phys. Rev. Lett. 101, 107006 (2008).

${ }^{5}$ P. Cheng, B. Shen, G. Mu, X. Zhu, F. Han, B. Zeng, and H.-H. Wen, EPL 85, 67003 (2009).

${ }^{6}$ R. Mittal, Y. Su, S. Rols, T. Chatterji, S. L. Chaplot, H. Schober, M. Rotter, D. Johrendt, and Th. Brueckel, Phys. Rev. B 78, 104514 (2008).

${ }^{7}$ R. Mittal, M. Zbiri, S. Rols, Y. Su, Y. Xiao, H. Schober, S. L. Chaplot, M. Johnson, T. Chatterji, S. Matsuishi, H. Hosono, and Th. Brueckel, Phys. Rev. B 79, 214514 (2009).

${ }^{8}$ A. D. Christianson, E. A. Goremychkin, R. Osborn, S. Rosenkranz, M. D. Lumsden, C. D. Malliakas, I. S. Todorov, H. Claus, D. Y. Chung, M. G. Kanatzidis, R. I. Bewley, and T. Guidi, Nature (London) 456, 930 (2008).

${ }^{9}$ C. de la Cruz, Q. Huang, J. W. Lynn, J. Li, W. Ratcliff II, J. L. Zarestky, H. A. Mook, G. F. Chen, J. L. Luo, N. L. Wang, and P. C. Dai, Nature (London) 453, 899 (2008).

${ }^{10}$ J. Zhao, Q. Huang, C. de la Cruz, S. Li, J. W. Lynn, Y. Chen, M. A. Green, G. F. Chen, G. Li, Z. Li, J. L. Luo, N. L. Wang, and P. Dai, Nature Mater. 7, 953 (2008).

${ }^{11}$ Q. Huang, Y. Qiu, W. Bao, M. A. Green, J. W. Lynn, Y. C. Gasparovic, T. Wu, G. Wu, and X. H. Chen, Phys. Rev. Lett. 101, 257003 (2008).

${ }^{12}$ Y. Su, P. Link, A. Schneidewind, Th. Wolf, P. Adelmann, Y. Xiao, M. Meven, R. Mittal, M. Rotter, D. Johrendt, Th. Brueckel, and M. Loewenhaupt, Phys. Rev. B 79, 064504 (2009).

${ }^{13}$ I. I. Mazin, D. J. Singh, M. D. Johannes, and M. H. Du, Phys. Rev. Lett. 101, 057003 (2008).

${ }^{14}$ T. Yildirim, Phys. Rev. Lett. 101, 057010 (2008).

${ }^{15}$ Q. M. Si and E. Abrahams, Phys. Rev. Lett. 101, 076401 (2008).

${ }^{16}$ C. Fang, H. Yao, W. F. Tsai, J. P. Hu, and S. A. Kivelson, Phys. Rev. B 77, 224509 (2008).
${ }^{17}$ I. I. Mazin and M. D. Johannes, Nat. Phys. 5, 141 (2009).

${ }^{18} \mathrm{~S}$. Matsuishi, Y. Inoue, T. Nomura, M. Hirano, and H. Hosono, J. Phys. Soc. Jpn. 77, 113709 (2008).

${ }^{19}$ M. Tegel, S. Johansson, V. Weiss, I. Schellenberg, W. Hermes, R. Poettgen, and D. Johrendt, EPL 84, 67007 (2008).

${ }^{20}$ F. Han, X. Zhu, G. Mu, P. Cheng, and H.-H. Wen, Phys. Rev. B 78, 180503(R) (2008).

${ }^{21}$ J. Rodríguez-Carvajal, Physica B 192, 55 (1993).

${ }^{22}$ Y. Xiao, Y. Su, R. Mittal, T. Chatterji, T. Hansen, C. M. N. Kumar, S. Matsuishi, H. Hosono, and Th. Brueckel, Phys. Rev. B 79, 060504(R) (2009).

${ }^{23}$ S. G. Brush, Rev. Mod. Phys. 39, 883 (1967).

${ }^{24}$ P. J. Baker, I. Franke, T. Lancaster, S. J. Blundell, L. Kerslake, and S. J. Clarke, Phys. Rev. B 79, 060402(R) (2009).

${ }^{25}$ A. I. Goldman, D. N. Argyriou, B. Ouladdiaf, T. Chatterji, A. Kreyssig, S. Nandi, N. Ni, S. L. Bud'ko, P. C. Canfield, and R. J. McQueeney, Phys. Rev. B 78, 100506(R) (2008).

${ }^{26}$ A. Jesche, N. Caroca-Canales, H. Rosner, H. Borrmann, A. Ormeci, D. Kasinathan, H. H. Klauss, H. Luetkens, R. Khasanov, A. Amato, A. Hoser, K. Kaneko, C. Krellner, and C. Geibel, Phys. Rev. B 78, 180504(R) (2008).

${ }^{27}$ Y. Luo, Q. Tao, Y. Li, X. Lin, L. Li, G. Cao, Z.-A. Xu, Y. Xue, H. Kaneko, A. V. Savinkov, H. Suzuki, C. Fang, and J. Hu, Phys. Rev. B 80, 224511 (2009).

${ }^{28}$ G. Wu, Y. L. Xie, H. Chen, M. Zhong, R. H. Liu, B. C. Shi, Q. J. Li, X. F. Wang, T. Wu, Y. J. Yan, J. J. Ying, and X. H. Chen, J. Phys.: Condens. Matter 21, 142203 (2009).

${ }^{29}$ K. Ishida, Y. Nakai, and H. Hosono, J. Phys. Soc. Jpn. 78, 062001 (2009).

${ }^{30}$ D. C. Wallace, Thermodynamics of Crystals (Dover, New York, 1998).

${ }^{31}$ R. W. Grosse-Kunstleve and P. D. Adams, J. Appl. Crystallogr. 35, 477 (2002).

${ }^{32}$ B. C. Sales, B. C. Chakoumakos, D. Mandrus, and J. W. Sharp, J. Solid State Chem. 146, 528 (1999).

${ }^{33}$ R. Mittal, M. Zbiri, S. Rols, Y. Su, Y. Xiao, H. Schober, S. Chaplot, M. Johnson, T. Chatterji, Y. Inoue, S. Matsuishi, H. Hosono, and T. Brueckel, arXiv:0912.1782 (unpublished).

${ }^{34}$ I. A. Nekrasov, Z. V. Pchelkina, and M. V. Sadovskii, JETP Lett. 88, 679 (2008). 